\title{
Correlation of Lipid Profile Levels in Young Smokers and Nonsmokers with Special Reference to Coronary Artery Disease
}

\author{
Mamatha B Patil ${ }^{1}$, Jeslin V James ${ }^{2}$, Bingi Somanath ${ }^{3}$
}

\begin{abstract}
Cigarette smoking is a major cause of cardiovascular disease and is responsible for approximately 140,000 premature deaths from cardiovascular diseases each year. Cigarette smoke delivers a high concentration of oxidizing chemicals and exposure to these chemicals is associated to depletion of endogenous levels of antioxidants, which contribute to a number of the potential mechanism of cardiovascular disease. Altered blood coagulation, impaired integrity of the arterial wall, and changes in blood lipid and lipoprotein concentrations predisposes to coronary artery disease $(C A D)$ in smokers.

Aims and objective: To compare lipid profile in smokers and nonsmokers. Determine dose-dependent relationship, durational significance between smoking and lipid profile among smokers. Evaluation for CAD in both smokers and nonsmokers.

Materials and methods: The study was carried out in 100 healthy male smokers and 100 healthy male nonsmokers selected from volunteers from RajaRajeswari Medical College, Bengaluru. All patients were subjected to fasting lipid profile, cardiac evaluation by doing ECG/ECHO/ TMT in smokers and nonsmokers.

Results: The mean duration of smoking was 6.6 years. The mean level of total cholesterol, LDL cholesterol, and triglycerides is significantly increased ( $p$ value $>0.001$ ), whereas the mean level of $\mathrm{HDL}$ cholesterol is significantly low ( $p$ value $>0.001$ ) in smokers compared to nonsmokers. There is a direct relationship exist between the severity of smoking and an increase in serum lipids. We have observed ECHO and TMT changes in smokers who have smoked for a duration of $>6$ years.

Conclusion: We have observed that there is a direct relationship exists between the severity of smoking and an increase in the serum lipid profile levels as well as greater risk for the development of coronary heart disease. So, it is strongly recommended to avoid smoking for the benefit of cardiac health.

Keywords: Cigarette smoking, Coronary artery disease, Dyslipidemia, Lipid profile.

The Journal of Medical Sciences (2020): 10.5005/jp-journals-10045-00150
\end{abstract}

\section{INTRODUCTION}

Smoking represents an important and rapidly growing global cause of cardiovascular mortality worldwide. Evidence since the early 1950s indicates that $>25$ diseases are now known or strongly suspected to be related to smoking. WHO estimated that unless the current smoking pattern is reversed, tobacco will be responsible for 10 million deaths per year, by $2020-2030$ with $70 \%$ of deaths occurring in developing countries.

In India, tobacco kills 8-10 lakhs of people each year and many of these deaths will occur in very young people. It has been estimated that an average of five-and-a-half minutes, a life is lost. Deaths attributable to tobacco are expected to rise from $1.4 \%$ of all deaths in 1990 to $13.3 \%$ in 2020. India, as per WHO, will have the highest rate of rising in tobacco-related deaths during this period compared with all other countries/regions. ${ }^{1}$ Youth in general and adolescents in particular fall prey to this deadly habit.

Cigarette smoking is one of the major modifiable risk factors of cardiovascular disease, in the form of atherosclerosis and coronary artery disease (CAD), it accelerates the atherosclerotic process by its proatherosclerotic and prothrombotic actions. Acute events such as ventricular fibrillation and sudden cardiac death are also increased by smoking. ${ }^{2}$

Although smoking has been established as an independent risk factor for coronary heart disease, the mechanism by which it increases the risk of coronary heart disease are modifiable ones like
1,3 Department of Internal Medicine, RajaRajeswari Medical College and Hospital, Bengaluru, Karnataka, India

${ }^{2}$ Department of Critical Care, Nirmala Hospital, Kozhikode, Kerala, India Corresponding Author: Mamatha B Patil, Department of Internal Medicine, RajaRajeswari Medical College and Hospital, Bengaluru, Karnataka,India,Phone:+91 9845680586,e-mail:dr.mamatharamesh@ yahoo.in

How to cite this article: Patil MB, James JV, Somanath B. Correlation of Lipid Profile Levels in Young Smokers and Nonsmokers with Special Reference to Coronary Artery Disease. J Med Sci 2020;6(2):23-27.

Source of support: Nil

Conflict of interest: None

hypertension, dyslipidemia, diabetes mellitus, changing lifestyle, and non-modifiable ones like age and sex.

The mechanism by which it increases the risk of coronary heart disease is unclear. ${ }^{3}$

- Nicotine from a cigarette may induce arrhythmias.

- Increased CO in the blood of cigarette smokers may damage the endothelium and accelerate the entry of cholesterol into the wall of the artery promoting the development of atherosclerosis and thrombosis.

- Smoking enhances platelet aggregation, oxidants from smoking damage vascular endothelium. 
- An additional mechanism has been recently suggested that smoking adversely affects the concentration of plasma lipids and lipoproteins.

- In some studies, smokers had increased plasma cholesterol levels, in others plasma cholesterol levels been lower. ${ }^{4}$ Only a few studies have specifically examined the plasma lipoproteins according to smoking status or the number of cigarettes (dosage). Smokers are reported to have high LDL and low HDL cholesterol levels than nonsmokers. $^{5}$

- Thus, the need of the hour is timely intervention through smoking cessation. Our study was carried out in young smokers to demonstrate the effects of smoking on lipid profile and evaluation of $C A D$, and the potential benefits of primordial prevention from CAD in such populations.

\section{Materials and Methods}

The study was carried out in 100 healthy male smokers and 100 healthy male nonsmokers selected from volunteers who had attended medicine OPD as well as IPD patients at RRMCH Bengaluru.

\section{Inclusion Criteria}

All the male patients (smokers and nonsmokers) are in the age group of $18-40$ years. Subjects were divided into four groups based on smoking habits as nonsmokers: (never smoked/left smoking for 5 years), mild smokers: (1-10 cigarettes/day at least 2 years), moderate smokers: (11-20 cigarettes/day at least 3-5 years), and heavy smokers: ( $>20$ cigarettes/day $>5$ years).

\section{Exclusion Criteria}

Patients with comorbidities viz., T2DM, HTN, dyslipidemia, chronic alcoholism, and who are on lipid-lowering agents, beta-blockers, diuretics were excluded from the study. Patients who have not given consent for the study.

Detailed history and physical examination along with systemic examination were done in all the patients and submitted for the fasting lipid profile, ECG, ECHO, and TMT.

\section{Statistical Analysis}

Results were analyzed by using suitable statistical software (SPSS version 23). Chi-square/Fischer's exact test has been used to find the significance of study parameters.

\section{Type of Study}

Cross-sectional observational study.

\section{Results}

Fifty-five percent of the smokers in the age group of 21-30 years and $44 \%$ of the patients were in $31-40$ years (Table 1 and Fig. 1). Sixty-five percent of the patients were smokers for $>3-5$ years

Table 1: Age distribution among nonsmokers and smokers

\begin{tabular}{lrcrrr}
\hline & \multicolumn{3}{c}{ Nonsmokers } & & \multicolumn{2}{c}{ Smokers } \\
\cline { 2 - 3 } \cline { 5 - 6 } Age in years & No. & \multicolumn{1}{c}{$\%$} & & No. & \multicolumn{1}{c}{$\%$} \\
\hline$<20$ & 1 & 1 & & 1 & 1.0 \\
$21-30$ & 53 & 55 & & 55 & 55.0 \\
$31-40$ & 46 & 44 & & 44 & 44.0 \\
Total & 100 & 100.0 & & 100 & 100.0 \\
Mean \pm SD & & $31.29 \pm 5.71$ & & & $31.22 \pm 5.13$ \\
\hline
\end{tabular}

(Table 2). Forty-two percent of the patients were mild smokers, $44 \%$ of the patient were moderate smokers (Table 3). Forty-six percent of the smokers were having $\mathrm{HDL}$ cholesterol $<35 \mathrm{mg} / \mathrm{dL}$, only $5 \%$ patients of nonsmokers were having $<35 \mathrm{mg} / \mathrm{dL}, 91 \%$ of smokers, $98 \%$ of the nonsmokers had LDL $c$ in the range of $70-190$ $\mathrm{mg} / \mathrm{dL}, 43 \%$ of smokers, $11 \%$ of the nonsmokers had triglycerides levels in the range of $150-500 \mathrm{mg} / \mathrm{dL}$ (Table 4). Thirty-eight percent of the smokers, $15 \%$ of the nonsmokers had serum cholesterol in the range of $200-280 \mathrm{mg} / \mathrm{dL}$ (Table 5). The mean level of HDL was lowest in heavy smokers, the mean level of LDL cholesterol was highest in heavy smokers, the mean level of triglycerides was high in heavy smokers, the mean level of total c was high in heavy smokers (Table 6), 42 (21\%) of the smokers had LVH, whereas 158 (79\%) had normal echo findings (Fig. 2). Twenty-two patients had TMT positive (moderate and heavy smokers) (Fig. 3 and Table 7). Comparative studies with our study (Table 8).

\section{Discussion}

Cigarette smokers have a higher risk of CAD than nonsmokers. Several possible explanations have been offered for this association, including altered blood coagulation, impaired integrity of the arterial wall, and changes in blood lipid and lipoprotein concentrations. Smoking in different forms is a major risk factor for atherosclerosis and coronary heart disease.

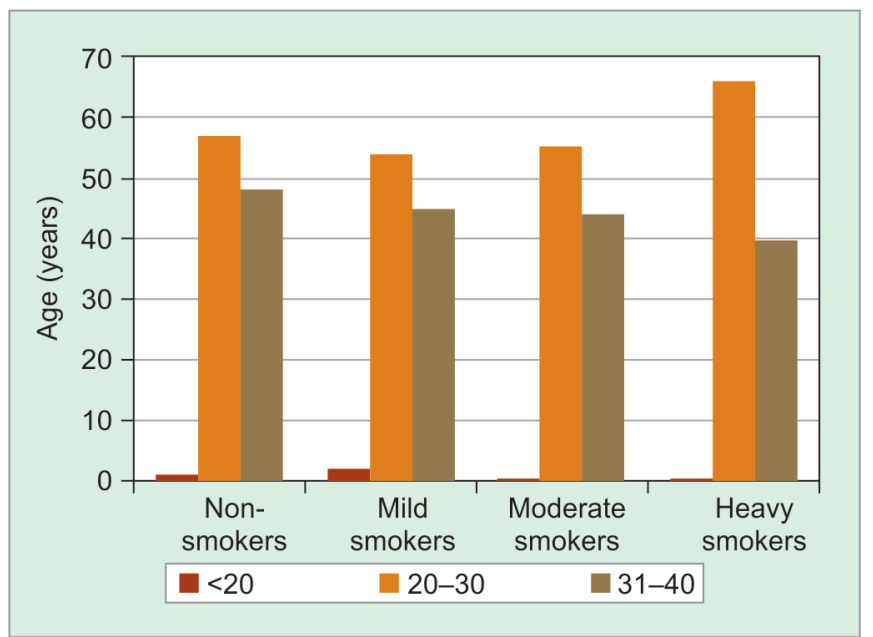

Fig. 1: Age distribution of patients studied in relation to severity of smoking with nonsmokers

Table 2: Distribution of patients according to years of smoking

\begin{tabular}{lcc}
\hline Year of smoking & No. of patients & $\%$ \\
\hline$>2$ & 9 & 9 \\
$3-5$ & 65 & 65 \\
$6-10$ & 26 & 26 \\
Total & 100 & 100 \\
\hline
\end{tabular}

Table 3: No. of cigarettes/day in cases studied

\begin{tabular}{lcc}
\hline No. of cigarettes/day & No. of patients & $\%$ \\
\hline$<10$ & 42 & 42.0 \\
$11-20$ & 44 & 44.0 \\
$>20$ & 14 & 14.0 \\
Total & 100 & 100.0 \\
\hline
\end{tabular}


Table 4: Lipid profile in nonsmokers and smokers

\begin{tabular}{|c|c|c|c|c|c|c|}
\hline \multirow[b]{2}{*}{ Lipid profile } & \multicolumn{3}{|c|}{ Nonsmokers $(n=100)$} & \multicolumn{2}{|c|}{ Smokers $(n=100)$} & \multirow[b]{2}{*}{$p$ value } \\
\hline & & No & $\%$ & No & $\%$ & \\
\hline \multicolumn{7}{|c|}{ High density lipoprotein (mg/dL) } \\
\hline$<35$ & 5 & & 5.0 & 46 & 46.0 & $<0.001$ \\
\hline $35-60$ & 95 & & 95.0 & 54 & 54.0 & \\
\hline$>60$ & 0 & & 0.0 & 0 & 0.0 & \\
\hline \multicolumn{7}{|c|}{ Low density lipoproteins (mg/dL) } \\
\hline$<70$ & 1 & & 1.0 & 6 & 6.0 & $<0.002$ \\
\hline $70-190$ & 98 & & 98.0 & 91 & 91.0 & \\
\hline$>190$ & 1 & & 1.0 & 3 & 3.0 & \\
\hline \multicolumn{7}{|c|}{ Triglycerides (mg/dL) } \\
\hline$<150$ & 89 & & 89.0 & 57 & 57.0 & $<0.001$ \\
\hline $150-500$ & 11 & & 11.0 & 43 & 43.0 & \\
\hline$>500$ & 0 & & 0.0 & 0 & 0.0 & \\
\hline
\end{tabular}

Table 5: Serum cholesterol in smokers and nonsmokers

\begin{tabular}{lrrrrr}
\hline & \multicolumn{3}{c}{ Nonsmokers } & & \multicolumn{2}{c}{ Smokers } \\
\cline { 2 - 3 } \cline { 5 - 6 } Serum cholesterol & No & $\%$ & & No & $\%$ \\
\hline$<200$ & 85 & 85.0 & & 61 & 61.0 \\
$200-280$ & 15 & 15.0 & & 38 & 38.0 \\
$>280$ & 0 & 0.0 & & 0 & 0.0 \\
Total & 100 & 100.0 & & 100 & 100.0 \\
Mean \pm SD & & $169.37 \pm 2.73$ & & & $186.03 \pm 32.80$ \\
\hline
\end{tabular}

Table 6: A study on lipid profile of patients studied in relation to the severity of smoking with nonsmokers

\begin{tabular}{lcccccc}
\hline Lipid profile & Nonsmokers & Mild-smokers & Moderate-smokers & Heavy-smokers & Total & $p$ value \\
\hline $\begin{array}{l}\text { High-density } \\
\text { lipoprotein }(\mathrm{mg} / \mathrm{dL})\end{array}$ & $39.62 \pm 4.24$ & $35.37 \pm 6.70$ & $34.68 \pm 4.87$ & $37.71 \pm 5.50$ & $37.51 \pm 5.50$ & $<0.001$ \\
$\begin{array}{l}\text { Low-density } \\
\text { lipoprotein }(\mathrm{mg} / \mathrm{dL})\end{array}$ & $120.42 \pm 27.33$ & $122.70 \pm 35.14$ & $133.38 \pm 40.58$ & $134.00 \pm 42.44$ & $124.70 \pm 33.65$ & $<0.002$ \\
$\begin{array}{l}\text { Triglycerides }(\mathrm{mg} / \mathrm{dL}) \\
\text { Serum cholesterol }\end{array}$ & $108.99 \pm 30.33$ & $131.10 \pm 40.55$ & $144.77 \pm 51.24$ & $151.79 \pm 47.13$ & $124.50 \pm 42.21$ & $<0.001$ \\
Mean \pm SD & $169.37 \pm 24.73$ & $182.69 \pm 33.71$ & $184.74 \pm 32.62$ & $200.00 \pm 28.99$ & $177.66 \pm 30.14$ & $<0.001$ \\
\hline
\end{tabular}

ECG does not show ischemic changes, tachycardia, abnormal rhythm, chamber hypertrophy

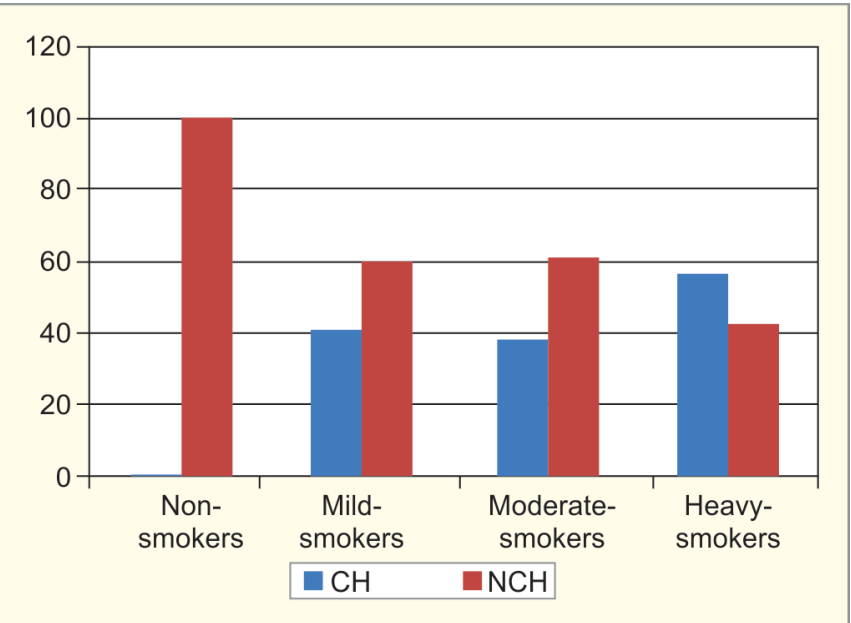

Fig. 2: Echocardiography findings of patients studied in relation to the severity of smoking with nonsmokers. $\mathrm{CH}$, changes; $\mathrm{NCH}$, non-changes (changes - concentric LVH, pulmonary hypertension, RVH)

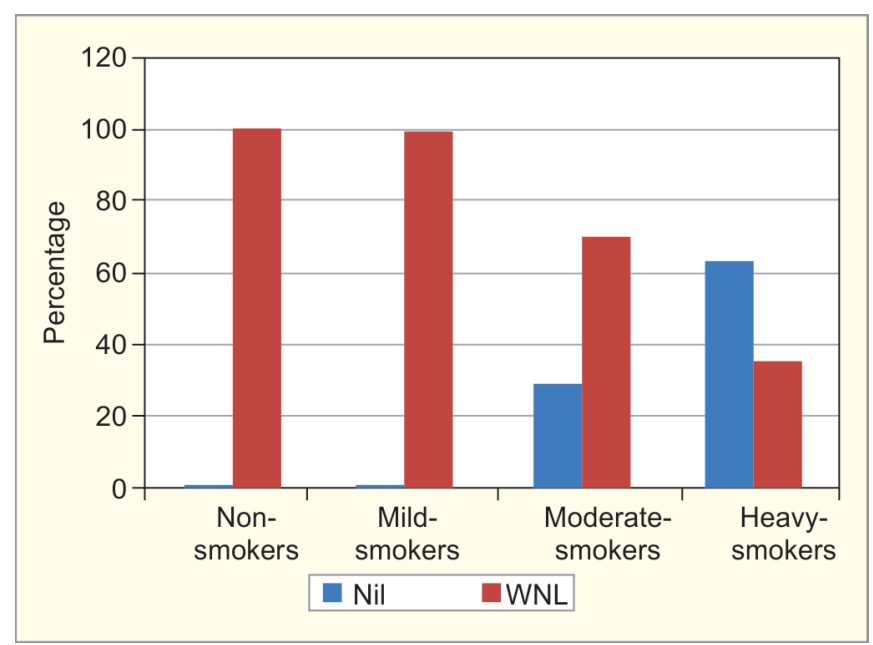

Fig. 3: Graphical representation of treadmill test findings of patients studied in relation to the severity of smoking with nonsmokers 
Correlation of Lipid Profile Levels in Young Smokers and Nonsmokers with Special Reference to CAD

Table 7: Treadmill test findings of patients studied in relation to the severity of smoking with nonsmokers

\begin{tabular}{lccccc}
\hline Treadmill test & Nonsmokers & Mild smokers & Moderate smokers & Heavy smokers & Total \\
\hline Positive & $0(0 \%)$ & $0(0 \%)$ & $13(29.5 \%)$ & $9(64.3 \%)$ & $22(11 \%)$ \\
WN L & $100(100 \%)$ & $42(100 \%)$ & $31(70.5 \%)$ & $5(35.7 \%)$ & $178(89 \%)$ \\
Total & $100(100 \%)$ & $42(100 \%)$ & $44(100 \%)$ & $14(100 \%)$ & $200(100 \%)$ \\
\hline
\end{tabular}

Fisher's exact test

Table 8: Comparison of lipid profiles of smokers from various studies

\begin{tabular}{|c|c|c|c|c|c|c|c|c|c|c|c|}
\hline \multirow[b]{2}{*}{ Author } & & \multicolumn{2}{|c|}{ Mean TC } & \multicolumn{2}{|c|}{ Mean TG } & \multicolumn{2}{|c|}{ Mean HDL } & \multicolumn{2}{|c|}{ Mean LDL } & \multirow[t]{2}{*}{ TMT } & \multirow[t]{2}{*}{ Echo } \\
\hline & & NS & SMO & NS & $S M O$ & NS & SMO & NS & $S M O$ & & \\
\hline Neki & NS-31 SMO-30 & 164.1 & 181.0 & 129 & 173.0 & 46.6 & 43.8 & 87.0 & 103.7 & - & \\
\hline Hemachandran et al. & NS-50 SMO-50 & 163 & 181 & 100 & 113 & 43 & 41.21 & 100 & 117.71 & - & \\
\hline Sreenivasa Rao et al. & NS-25 SMO-25 & 141 & 163 & 92 & 117 & 60 & 54 & 84 & 113 & - & \\
\hline Singha et al. & NS-50 SMO-50 & 195 & 227 & 111 & 145 & 40 & 37 & 128 & 161 & - & \\
\hline Prathamesh H Kamble & NS-100 SMO-100 & 145 & 228 & 72 & 113 & 46 & 29.14 & 102 & 159.26 & - & \\
\hline Present study & NS-100 SMO-100 & 169 & 200 & 108.99 & 151.79 & 39.6 & 30.7 & 120 & 134 & $22 \%$ & $42 \%$ \\
\hline
\end{tabular}

Compared with western society, cigarette smoking is probably occurring in the third decade of life in Indian society accounting for the maximum number of smokers. During the student life, the late adolescence, and when they bloom out to college life, they get captivated by the "SMOKE FREE". Few start smoking because they feel like adults, with a cigarette stuck to their lips and the smoke emanating from their nostrils. A majority would quit smoking, while a minor group would succumb to more addictive habits like ganja. In India, the social class determines the type of smoking; beedi costing less makes a manual labor smoke, while filter cigarettes are used by the elite group. At this juncture, the wise man's word is worth remembering-A cigarette is like a roll of paper with fire at one end and a fool at the other end. In the present study, 100 smokers and 100 nonsmokers were studied for their lipid profile, age, sex, obesity, alcohol, diet-these parameters were matched in smokers and nonsmokers. In India, female smokers are few; for a majority, it is a "taboo". Hence, this study is particularly comprised of male smokers. The observed values in the present study are in concordance with the data published in India and abroad.

It is revealed that triglycerides, LDL, VLDL, HDL, and TC were statistically significantly higher in smokers as compared to nonsmokers. The mean serum total cholesterol was higher in smokers when compared to nonsmokers, which was statistically significant $(p<0.05)$. The mean serum triglycerides were higher in smokers when compared to nonsmokers, which was statistically significant $(p<0.05)$. The mean serum LDL was higher in smokers when compared to nonsmokers, which was statistically significant $(p<0.05)$. The mean serum HDL was higher in nonsmokers when compared to smokers, which was statistically significant $(p<0.05)$.

Several studies have shown an association between cigarette smoking and altered lipid profile and lipoprotein concentrations, but many of these lacked enough statistical power to establish a firm association. By combining the results of individual studies in the present analysis, we have shown conclusively that smoking is associated with significantly higher serum concentrations of total cholesterol, triglycerides, and LDL and lower serum concentrations of HDL and that this association with dose-dependent and evaluation of CAD by doing TMT. To our knowledge, the data relevant to changes in serum lipid and lipoprotein concentrations associated with a degree of exposure to cigarette smoke have not previously been compiled and reviewed. The dose-dependent relation of coronary evaluation that we found may provide new evidence for casual relation.

The increase in the concentration of serum total cholesterol, triglycerides, LDL, VLDL, and fall in the levels of anti-atherogenic $\mathrm{HDL}$, have been reported from various studies. ${ }^{6-8}$ A study conducted by $\mathrm{Nesi}^{8}$ from North India reported mean serum TG levels in smokers were significantly high, as compared to nonsmokers. The mean serum HDL was significantly lower in chronic smokers as compared to nonsmokers. This is comparable to the present study.

In the present study, mean serum triglycerides level was found to be $140.01 \pm 46.65$ in smokers and $108.99 \pm 30.33$ in nonsmokers. Among the nonsmokers, serum HDL level was found to be $39.62 \pm$ 4.24 , whereas in smokers HDL level was $35.39 \pm 5.82$. The association between hypertension and increased level LDL was observed in a South Indian study conducted by Chandala et al. ${ }^{8}$ In our study, LDL level was comparatively higher among smokers.

A similar study conducted by Chandran et al. ${ }^{9}$ showed that smoking alters the lipid profile adversely causing dyslipidemia in smokers and the change becomes more marked with the number of cigarettes smoked. In our study, $14 \%$ of the subjects were heavy smokers ( $>20$ cigarettes per day). The serum cholesterol level was found to be higher in heavy smokers in our study. About $50 \%$ of heavy smokers were with a serum cholesterol level of 200-280 $\mathrm{mg} / \mathrm{dL}$. Another Indian study from Karnataka by Afshan et al. has reported total cholesterol, HDL level was lower in subjects who were smoking $>10$ cigarettes per day. This is similar to the present study where the HDL level was lower among moderate smokers. About $59 \%$ of moderate smokers showed serum cholesterol levels $<200 \mathrm{mg} / \mathrm{dL}$. A study conducted by Khan et al. ${ }^{10}$ from Tamil Nadu reported that smoking 5-6 cigarettes for a duration of 5 years has no significant impact on cholesterol and triglyceride levels of the test volunteers.

Another Indian study by Sharma et al. ${ }^{11}$ has reported the role of smoking in the causation of premature atherosclerosis in young males in the age group of $30-40$ years, with $8 \%$ of smokers showed 
evidence of P Pulmonale on ECG. In the present study conducted, $45 \%$ of smokers were in the age group of 31-40 years. Decreased $\mathrm{HDL}$ and increased LDL level were observed in a study conducted by Singha et al. on smokers of the age group 25-35 years. A similar study from Nagpur by Prathamesh H Kamble ${ }^{12}$ on tobacco users (smokers and smokeless tobacco users) showed significantly higher lipid profile values than controls. The lipid profile value of smokers was higher compared to controls in our study.

A study from South India by Sreenivasa Rao et al. ${ }^{13}$ has found that the prevalence of CAD was higher among smokers than nonsmokers, which is in agreement with the results of our study. The present study showed significantly higher triglyceride levels and significantly lower HDL levels and positive TMT among smokers.

Cigarette smoking is an established risk factor for cardiovascular disease in young subjects. Tobacco smoking, elevated LDL, TGs, and hypertension lead to CAD. In the present study, $54 \%$ of smokers were in the age group of 20-30 years. The serum cholesterol level among them was found to be $200-280 \mathrm{mg} / \mathrm{dL}$. Thus, early detection of dyslipidemia is necessary among smokers to prevent complications associated with smoking.

\section{CONCLUSION}

We have observed that there is a direct relationship exists between the severity of smoking and an increase in the serum lipid profile levels as well as greater risk for the development of coronary heart disease. So, it is strongly recommended to avoid smoking for the benefit of cardiac health in young smokers.

\section{Acknowledgment}

Dr Debarchana Sarkar, 1st year PG student, Department of General Medicine, RRMCH.

\section{References}

1. Ball K. Smoking spells death for millions. World Health Forum 1986;7:211-216.

2. Baura RS, Ambrose JA, Eales-Reynolds L-J, et al. Heavy and light cigarette smokers have similar dysfunction of endothelial vasoregulatory activity. J Am Coll Cardiol 2002;39(11):1758-1763.

3. Yeolekar ME. Coronary Heart Disease Risk factors known and the less known journal of Association of Physicians of India vol. 51 october 2003.

4. 3rd report of the national educational programme expert panel on detection, evaluation and treatment of high blood cholesterol in adults. Archinternal medicine 2008: pp. 1-41.

5. Pershell SD, Liod Jones DM, Baker DW. Implication of changing national education programme, cholesterol goals for the treatment and control of hypocholesterolemia. J Gen Int Med 2006;21(2):171176. DOI: 10.1007/s11606-006-0253-2.

6. Ambrose JA, Barua RS. The pathophysiology of cigarette smoking and cardiovascular disease: an update. J Am Coll Cardiol 2004;43(10):17311737. DOI: 10.1016/j.jacc.2003.12.047.

7. Neki NS. Lipid profile in chronic smokers - a clinical study. JIACM 2002;3(1):51-54.

8. Chandala SR, Kilim SR. Plasma lipid profile in chronic tobacco smokers and hypertensives. IOSR J Pharm Biolog Sci 2014;9(6):55-57.

9. Dussa HCK, Parchwani D, Maheria P. Status of lipid profile in active smokers. Int J Res Med 2012;1(1):17-20.

10. Khan I, Farhana M, Sai Ramesh AA, et al. Effects of smoking on serum lipid levels in nascent young Indian smokers. Int J Pharma Sci Res (IJPSR) 2012;3(9):463-466.

11. Sharma SB, Dwivedi S, Prabhu KM, et al. Coronary risk variables in young asymptomatic smokers. Indian J Med Res 2005;122(3):205-210.

12. Kamble PH, Rode Mrunal MV, Phatak S, et al. Is smokeless tobacco use a risk factor for coronary artery disease? A comparative study of smokers and smokeless tobacco users. Indian J Bas Appl Med Res 2011;1(1):22-30.

13. Sreenivasa Rao $\mathrm{CH}$, Subashy E. The effect of chronic tobacco smoking and chewing on the lipid profile. J Clin Diagnos Res 2013;7(1):31-34. 